\title{
Two-Dimensional Materials as Catalysts for Energy Conversion
}

\author{
Samira Siahrostami ${ }^{1} \cdot$ Charlie Tsai $^{1,2} \cdot$ Mohammadreza Karamad $^{1}$. \\ Ralph Koitz ${ }^{2,3}$ - Max García-Melchor ${ }^{1,2} \cdot$ Michal Bajdich $^{2} \cdot$ Aleksandra Vojvodic $^{2}$. \\ Frank Abild-Pedersen ${ }^{2} \cdot$ Jens K. Nørskov ${ }^{1,2} \cdot$ Felix Studt $^{2,4,5}$
}

Received: 23 June 2016 / Accepted: 1 August 2016

(C) Springer Science+Business Media New York 2016

\begin{abstract}
Although large efforts have been dedicated to studying two-dimensional materials for catalysis, a rationalization of the associated trends in their intrinsic activity has so far been elusive. In the present work we employ density functional theory to examine a variety of two-dimensional materials, including, carbon based materials, hexagonal boron nitride $(h$-BN), transition metal dichalcogenides (e.g. $\mathrm{MoS}_{2}, \mathrm{MoSe}_{2}$ ) and layered oxides, to give an overview of the trends in adsorption energies. By examining key reaction intermediates relevant to the oxygen reduction, and oxygen evolution reactions we find that binding energies largely follow the linear scaling relationships observed for pure
\end{abstract}

Electronic supplementary material The online version of this article (doi:10.1007/s10562-016-1837-z) contains supplementary material, which is available to authorized users.

Felix Studt

felix.studt@kit.edu

1 SUNCAT Center for Interface Science and Catalysis, Department of Chemical Engineering, Stanford University, 443 Via Ortega, Stanford, CA 94305, USA

2 SUNCAT Center for Interface Science and Catalysis, SLAC National Accelerator Laboratory, 2575 Sand Hill Road, Menlo Park, CA 94025, USA

3 Department of Chemistry, University of Zurich, Winterthurerstrasse 190, 8057 Zurich, Switzerland

4 Institute of Catalysis Research and Technology, Karlsruhe Institute of Technology, Hermann-von-Helmholtz Platz 1, 76344 Eggenstein-Leopoldshafen, Germany

5 Institute for Chemical Technology and Polymer Chemistry, Karlsruhe Institute of Technology, Engesserstr. 18, 76131 Karlsruhe, Germany metals. This observation is very important as it suggests that the same simplifying assumptions made to correlate descriptors with reaction rates in transition metal catalysts are also valid for the studied two-dimensional materials. By means of these scaling relations, for each reaction we also identify several promising candidates that are predicted to exhibit a comparable activity to the state-of-the-art catalysts.

Graphical Abstract Scaling relationship for the chemisorption energies of $\mathrm{OH}^{*}$ and $\mathrm{OOH}^{*}$ on various $2 \mathrm{D}$ materials.

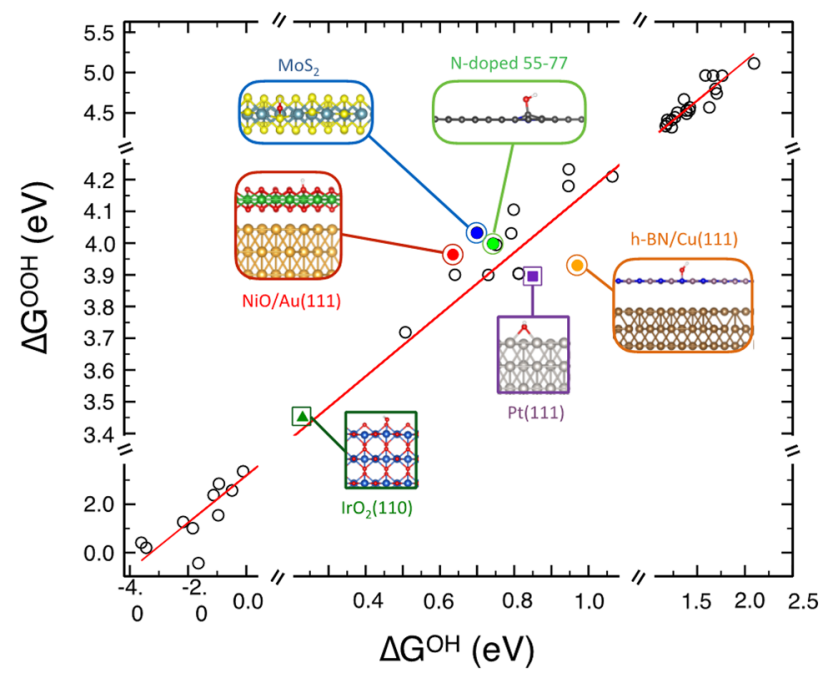

Inspired by the success of graphene in a broad range of electronic and energy applications [1-7], novel classes of layered two-dimensional (2D) materials have been intensively studied in the past few years [8-14]. Structurally modified graphene, hexagonal boron nitride $(h-\mathrm{BN})$, transition metal dichalcogenides (e.g. $\mathrm{MoS}_{2}, \mathrm{MoSe}_{2}$ ) and layered transition metal oxides, are some of the 2D materials that have found 
broad applications due to their high surface area to volume ratio, and desirable electrical, mechanical and thermal properties [15-18]. Because of their low cost and ability to form a wide range of nanostructures, these $2 \mathrm{D}$ materials are also being increasingly considered for electrochemical energy conversion and storage $[19,20]$, and they represent a promising class of alternatives to replace the commercial precious-metal catalysts [5-7].

In their pristine state, most 2D materials do not exhibit any chemical activity. However, their properties can be tailored by introducing dopants [19,21, 22], generating defects [23] and by supporting them on transition metals [24-26]. Nitrogen doped graphene, for instance, has been shown to catalyze the electrochemical reduction of oxygen to water and the oxygen reduction reaction, (ORR) [22, 27-34]. Other dopants such as boron [35], sulfur and phosphorous $[21,36]$ have been also proven to be interesting. Likewise, $h$ $\mathrm{BN}$, an otherwise inert material, becomes a promising ORR catalyst when supported on transition metals such as $\mathrm{Au}$ [24] and $\mathrm{Cu}$ [25]. Layered transition metal dichalcogenides, such as molybdenum sulfides and selenides, are active catalysts for the hydrogen evolution reaction (HER) and $\mathrm{CO}_{2}$ reduction reaction $[18,37-40]$, and their activity can be further improved by introducing defects and transition metal dopants [41-43]. Layered oxides based on earth-abundant transition metals such as nickel, iron, or cobalt, have been reported to be highly active materials for the oxygen evolution reaction (OER), specially the multimetallic oxides based on these elements [44-46]. Some of these catalysts have shown a comparable (or even better) performance in alkaline media than that of the state-of-the-art Ir and Ru based catalysts [47, 48], and therefore, they are particularly promising.

Recently, it has been shown that graphene based catalysts are able to catalyze both ORR and OER reactions [30-32]. This bifunctionality is particularly remarkable as it points out the great potential of these materials for a wide variety of applications. It is, however, not yet known whether other related two-dimensional materials exhibit the same versatility. Although the reaction conditions for the ORR and OER differ substantially, both processes involve the hydroxyl $\left(\mathrm{OH}^{*}\right)$ and hydroperoxo $\left(\mathrm{OOH}^{*}\right)$ intermediates, and the corresponding calculated chemisorption energies have been successfully employed to describe the ORR and OER reactivities [49, 50]. Furthermore, the energetics of these two intermediates have been found to be correlated through linear scaling relations on transition metal [51] as well as transition metal oxide surfaces [52], thus making it possible to establish volcano-type relationships for both ORR and OER [49, 53].

Herein, we seek to investigate trends in chemisorption energies for a wide range of different 2D materials (details and structures in Supplementary Information) in order to draw comparisons with currently known catalysts and evaluate their predicted activity for the ORR and OER reactions.
We find that the linear scaling relations between the adsorption energies of key intermediates of the ORR and OER reactions, i.e., $\mathrm{OH}^{*}$ and $\mathrm{OOH}^{*}$ [54], can be used to rapidly assess the catalytic activity of a material for ORR and OER. Our results also show that $2 \mathrm{D}$ materials represent an especially interesting class of catalysts offering a promising alternative to the commercial ones based on precious metals.

We employ density functional theory (DFT) calculations to study the chemisorption energies of $\mathrm{OH}^{*}$ and $\mathrm{OOH}^{*}$ on the selected 2D materials (SI). Calculations for the carbon based and chalcogenides were performed using the QUANTUM ESPRESSO program package [55] and the atomic simulation environment (ASE) [56]. The Bayesian Error Estimation Functional with van-der-Waals correction (BEEF-vdW) [57] was used, and the electronic wave functions were expanded in plane waves up to a cutoff energy of $500 \mathrm{eV}$, while the electron density was represented on a grid with an energy cutoff of $5000 \mathrm{eV}$. Core electrons were approximated with ultrasoft pseudopotentials. Calculations on the layered nickel oxide supported on gold $\left(\mathrm{NiO}_{\mathrm{x}} / \mathrm{Au}(111)\right)$ were performed using the Perdew-Burke-Ernzenhof (PBE) exchange correlation functional, as implemented in the VASP [58, 59]. For a better description of the $\mathrm{Co}(3 \mathrm{~d}), \mathrm{Ni}(3 \mathrm{~d})$ and $\mathrm{Ce}(4 \mathrm{f})$ electrons, the Hubbard effective terms $U_{e f f}(\mathrm{Co})=3.32 \mathrm{eV}$, $U_{\text {eff }}(\mathrm{Ni})=6.45 \mathrm{eV}$ and $U_{\text {eff }}(\mathrm{Ce})=4.5 \mathrm{eV}$ were added to the PBE functional through the rotationally invariant approach proposed by Dudarev et al. [60]. All periodic slab calculations were carried out using a plane wave kinetic energy cutoff of $500 \mathrm{eV}$ and a vacuum spacing of at least $15 \AA$. Further details on the slab setups and the $k$-point sampling used in calculations can be found in the references.

Although all materials studied herein present a 2D structure, their relevant active sites differ significantly. For graphene and $h$-BN, these sites are carbon and boron atoms, respectively. For $\mathrm{MoS}_{2}$ and $\mathrm{MoSe}_{2}$, the basal plane has been shown to be inert, and adsorbates bind to under-coordinated chalcogen atoms on the edges. On the other hand, for nickel and cobalt oxide films supported on gold $\left(\mathrm{NiO}_{\mathrm{x}} / \mathrm{Au}(111)\right)$, both edge and basal plane sites contribute to the OER activity [61].

Figure 1 displays the scaling relationship for the adsorption energies of the $\mathrm{OH}^{*}$ and $\mathrm{OOH}^{*}$ on the different studied 2D structures. The $\operatorname{Pt}(111)$ surface is depicted for comparison and the optimal ORR catalyst should have an $\mathrm{OH}^{*}$ binding energy approximately $0.2 \mathrm{eV}$ weaker than $\mathrm{Pt}(111)$ [53]. As can be seen in Fig. 1, all the computed adsorption energies follow the same scaling relation with.

$\Delta \mathrm{G}^{\mathrm{OOH}} \approx \Delta \mathrm{G}^{\mathrm{OH}}+3.2 \mathrm{eV}$

We note that this scaling relation is identical to that found for transition metal surfaces exposing the (111) facet [51, 


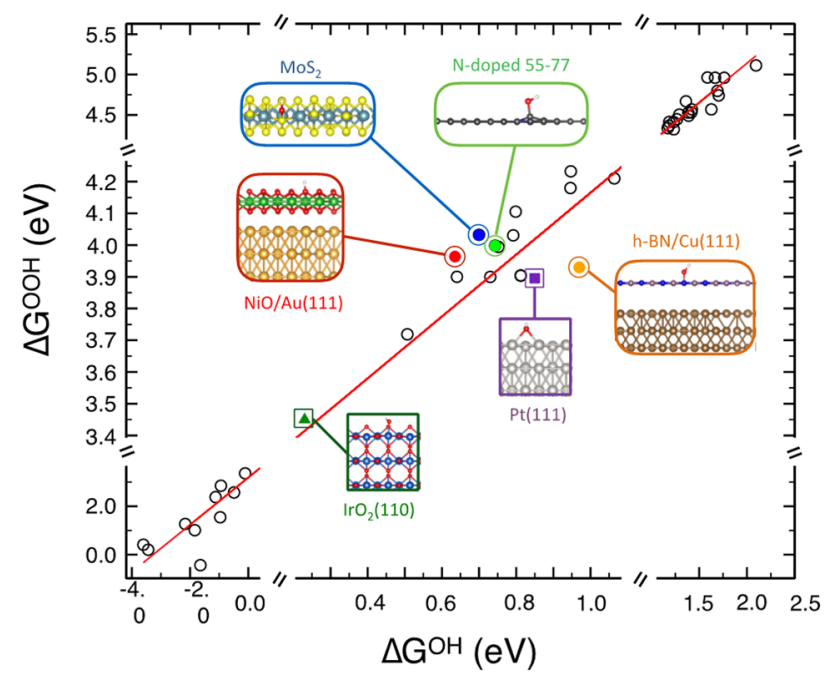

Fig. 1 Scaling relationship for the chemisorption energies of $\mathrm{OH}^{*}$ and $\mathrm{OOH}^{*}$ for various $2 \mathrm{D}$ materials. $\mathrm{Pt}(111)$ and $\mathrm{IrO}_{2}(110)$ data adapted from Refs. [49, 62] are shown for comparison in purple and dark green, respectively. $\mathrm{OH}$ and $\mathrm{OOH}$ adsorption energies are reported relative to liquid water and gas phase hydrogen using $\mathrm{H}_{2} \mathrm{O}(\mathrm{l})+{ }^{*} \rightarrow$ $\mathrm{OH}^{*}+1 / 2 \mathrm{H}_{2}(\mathrm{~g})$ and $2 \mathrm{H}_{2} \mathrm{O}(\mathrm{l})+* \rightarrow \mathrm{OOH}^{*}+3 / 2 \mathrm{H}_{2}(\mathrm{~g})$, respectively. $h$-BN/M and $\mathrm{NiO}_{\mathrm{x}} / \mathrm{Au}$ are adapted from Refs. $[25,61]$, respectively. Color code: C (gray), N (blue), O (red), H (white), B (pink), S (yellow), Mo (cyan), Cu (brown), Ni (green), Au (orange), Pt (silver), Ir (light blue)

$54,63]$ and similar to the values obtained for metal-oxides $[52,64]$. This linear scaling therefore suggests that the same descriptor based prediction of activity also holds for the 2D materials considered in this study. Interestingly, Fig. 1 also shows that there is a wide range of binding energies on the various materials, demonstrating the tunability of $2 \mathrm{D}$ materials. Thus, despite the wide variety of $2 \mathrm{D}$ materials, with different active sites and compositions, their scaling behavior is consistent with each other, and with the transition metals and metal oxides. This finding, however, also points to the fact that the herein studied 2D materials are unlikely to lead to major improvements in catalytic efficiency resulting from deviations from scaling relations.

Among all the investigated materials, we identify several 2D structures that have similar $\mathrm{OH}$ and $\mathrm{OOH}$ binding energies as $\mathrm{Pt}(111)$, and thus they are expected to be potential candidates for efficient ORR. These materials include the $\mathrm{N}$-doped version of Stone Wales defect in graphene (N-doped 55-77), $\mathrm{MoS}_{2}$, and $h-\mathrm{BN} / \mathrm{Cu}(111)$ (Fig. 1). In fact $h-\mathrm{BN} / \mathrm{Cu}(111)$ has been found to be a reasonably good catalysts for ORR [25], while $\mathrm{NiO}_{x} / \mathrm{Au}(111)$ has been both experimentally and theoretically proven to be active for OER [61]. The performance of this latter catalyst has been also shown to improve considerably through a synergistic effect that arises from using cerium as a dopant and gold as a metal support [61].

In summary, we have studied a large number of 2D materials involving a diverse range of chemical and geometrical structures and modifications, and showed that all the calculated adsorption energies follow the same scaling relations as established for transition metal surfaces. The existence of scaling relationships for these 2D materials suggests that the same simplifying assumptions used for predicting and evaluating new transition metal catalysts are also valid for this class of materials. Volcano-type analyses established for transition metals can thus be used for high-throughput screening of materials similar to those studied herein. Also important is the fact that the adsorption energies vary significantly depending on the type of active site, allowing for a wide range of possible activities, even within the same type of materials. For ORR some of the 2D-materials considered herein exhibit structures that provide adsorption energies similar to $\mathrm{Pt}$, one of the best ORR catalysts. However, the fact that the scaling relations for 2D materials are identical to those of pure metals, suggest that scaling limitations will also inhibit further improvements in their potential catalytic activity.

Acknowledgments We gratefully acknowledge support from the U.S. Department of Energy, Office of Sciences, Office of Basic Energy Sciences, to the SUNCAT Center for Interface Science and Catalysis. S.S and M.K acknowledge support from the Global Climate Energy Project (GCEP) at Stanford University (Fund No. 52454).

\section{References}

1. Geim AK, Novoselov KS (2007) The rise of graphene. Nat Mater 6:183-191. doi:10.1038/nmat1849

2. Geim AK (2009) Graphene: status and prospects. Science 324:1530-1534. doi:10.1126/science. 1158877

3. Neto AHC, Novoselov K (2011) New directions in science and technology: two-dimensional crystals. Rep Prog Phys 74:082501. doi:10.1088/0034-4885/74/8/082501

4. Bonaccorso F, Sun Z, Hasan T, Ferrari AC (2010) Graphene photonics and optoelectronics. Nat Photonics 4:611-622. doi:10.1038/nphoton.2010.186

5. Sun Y, Wu Q, Shi G (2011) Graphene based new energy materials. Energy Environ Sci 4:1113. doi:10.1039/c0ee00683a

6. Brownson DAC, Kampouris DK, Banks CE (2011) An overview of graphene in energy production and storage applications. J Power Sources 196:4873-4885. doi:10.1016/j.jpowsour.2011.02.022

7. Pumera M (2011) Graphene-based nanomaterials for energy storage. Energy Environ Sci 4:668-674. doi:10.1039/C0EE00295J

8. Gupta A, Sakthivel T, Seal S (2015) Recent development in 2D materials beyond graphene. Prog Mater Sci 73:44-126. doi:10.1016/j.pmatsci.2015.02.002

9. Koski KJ, Cui Y (2013) The new skinny in two-dimensional nanomaterials. ACS Nano 7:3739-3743. doi:10.1021/nn4022422

10. Chhowalla M, Shin HS, Eda G et al (2013) The chemistry of twodimensional layered transition metal dichalcogenide nanosheets. Nat Chem 5:263-275. doi:10.1038/nchem.1589

11. Das S, Robinson JA, Dubey $M$ et al (2015) Beyond graphene: progress in novel two-dimensional materials and van der Waals solids. Annu Rev Mater Res 45:1-27. doi:10.1146/ annurev-matsci-070214-021034

12. Kim SJ, Choi K, Lee B et al (2015) Materials for flexible, stretchable electronics: graphene and 2D materials. Annu Rev Mater Res 45:63-84. doi:10.1146/annurev-matsci-070214-020901 
13. Lotsch BV (2015) Vertical 2D heterostructures. Annu Rev Mater Res 45:85-109. doi:10.1146/annurev-matsci-070214-020934

14. Butler SZ, Hollen SM, Cao L et al (2013) Progress, challenges, and opportunities in two-dimensional materials beyond graphene. ACS Nano 7:2898-2926. doi:10.1021/nn400280c

15. Lee C, Wei X, Kysar JW, Hone J (2008) Measurement of the elastic properties and intrinsic strength of monolayer graphene. Science 321:385-388. doi:10.1126/science.1157996

16. Novoselov KS, Geim AK, Morozov SV et al (2004) Electric field effect in atomically thin carbon films. Science 306:666-669. doi:10.1126/science.1102896

17. Balandin AA, Ghosh S, Bao W et al (2008) Superior thermal conductivity of single-layer graphene. Nano Lett 8:902-907. doi:10.1021/n10731872

18. Kong D, Wang H, Cha JJ et al (2013) Synthesis of $\mathrm{MoS}_{2}$ and $\mathrm{MoSe}_{2}$ films with vertically aligned layers. Nano Lett 13:13411347. doi:10.1021/n1400258t

19. Dai L (2013) Functionalization of graphene for efficient energy conversion and storage. Acc Chem Res 46:31-42. doi:10.1021/ ar300122m

20. Wang H, Yuan X, Zeng G et al (2015) Three dimensional graphene based materials: Synthesis and applications from energy storage and conversion to electrochemical sensor and environmental remediation. Adv Colloid Interface Sci 221:41-59. doi:10.1016/j.cis.2015.04.005

21. Liu J, Song P, Ning Z, Xu W (2015) Recent advances in heteroatom-doped metal-free electrocatalysts for highly efficient oxygen reduction reaction. Electrocatalysis 6:132-147. doi:10.1007/ s12678-014-0243-9

22. Wong WY, Daud WRW, Mohamad AB et al (2013) Recent progress in nitrogen-doped carbon and its composites as electrocatalysts for fuel cell applications. Int J Hydrog Energy 38:9370-9386. doi:10.1016/j.ijhydene.2012.12.095

23. Terrones H, Lv R, Terrones M, Dresselhaus MS (2012) The role of defects and doping in 2D graphene sheets and 1D nanoribbons. Rep Prog Phys 75:062501. doi:10.1088/0034-4885/75/6/062501

24. Uosaki K, Elumalai G, Noguchi H et al (2014) Boron nitride nanosheet on gold as an electrocatalyst for oxygen reduction reaction: theoretical suggestion and experimental proof. J Am Chem Soc 136:6542-6545. doi:10.1021/ja500393g

25. Koitz R, Nørskov JK, Studt F (2015) A systematic study of metal-supported boron nitride materials for the oxygen reduction reaction. Phys Chem Chem Phys 17:12722-12727. doi:10.1039/ c5 $\mathrm{cp} 01384 \mathrm{~d}$

26. Feng L, Liu Y, Zhao J (2015) Iron-embedded boron nitride nanosheet as a promising electrocatalyst for the oxygen reduction reaction (ORR): a density functional theory (DFT) study. J Power Sources 287:431-438. doi:10.1016/j.jpowsour.2015.04.094

27. Ouyang W, Zeng D, Yu X et al (2014) Exploring the active sites of nitrogen-doped graphene as catalysts for the oxygen reduction reaction. Int J Hydrog Energy 39:15996-16005. doi:10.1016/j. ijhydene.2014.01.045

28. Qu L, Liu Y, Baek J-B, Dai L (2010) Nitrogen-doped graphene as efficient metal-free electrocatalyst for oxygen reduction in fuel cells. ACS Nano 4:1321-1326. doi:10.1021/nn901850u

29. Gong K, Du F, Xia Z et al (2009) Nitrogen-doped carbon nanotube arrays with high electrocatalytic activity for oxygen reduction. Science 323:760-764. doi:10.1126/science.1168049

30. Wang L, Yin F, Yao C (2014) N-doped graphene as a bifunctional electrocatalyst for oxygen reduction and oxygen evolution reactions in an alkaline electrolyte. Int J Hydrog Energy 39:1591315919. doi:10.1016/j.ijhydene.2014.04.071

31. Li M, Zhang L, Xu Q et al (2014) N-doped graphene as catalysts for oxygen reduction and oxygen evolution reactions: theoretical considerations. J Catal 314:66-72. doi:10.1016/j.jcat.2014.03.011
32. Zhang J, Zhao Z, Xia Z, Dai L (2015) A metal-free bifunctional electrocatalyst for oxygen reduction and oxygen evolution reactions. Nat Nanotechnol 10:444-452. doi:10.1038/nnano.2015.48

33. Liang W, Chen J, Liu Y, Chen S (2014) Density-functional-theory calculation analysis of active sites for four-electron reduction of $\mathrm{O}_{2}$ on $\mathrm{Fe} / \mathrm{N}$-doped graphene. ACS Catal 4:4170-4177. doi:10.1021/cs501170a

34. Studt F (2012) The oxygen reduction reaction on nitrogen-doped graphene. Catal Lett 143:58-60. doi:10.1007/s10562-012-0918-x

35. Fazio G, Ferrighi L, Di Valentin C (2014) Boron-doped graphene as active electrocatalyst for oxygen reduction reaction at a fuelcell cathode. J Catal 318:203-210. doi:10.1016/j.jcat.2014.07.024

36. Jiao Y, Zheng Y, Jaroniec M, Qiao SZ (2014) Origin of the electrocatalytic oxygen reduction activity of graphene-based catalysts: a roadmap to achieve the best performance. J Am Chem Soc 136:4394-4403. doi:10.1021/ja500432h

37. Hinnemann B, Moses PG, Bonde J et al (2005) Biomimetic hydrogen evolution: $\mathrm{MoS}_{2}$ nanoparticles as catalyst for hydrogen evolution. J Am Chem Soc 127:5308-5309. doi:10.1021/ ja0504690

38. Jaramillo TF, Jørgensen KP, Bonde J et al (2007) Identification of active edge sites for electrochemical $\mathrm{H}_{2}$ evolution from $\mathrm{MoS}_{2}$ nanocatalysts. Science 317:100-102. doi:10.1126/ science. 1141483

39. Chan K, Tsai C, Hansen HA, Nørskov JK (2014) Molybdenum sulfides and selenides as possible electrocatalysts for $\mathrm{CO}_{2}$ reduction. ChemCatChem 6:1899-1905. doi:10.1002/cctc.201402128

40. Bollinger MV, Lauritsen JV, Jacobsen KW et al (2001) Onedimensional metallic edge states in $\mathrm{MoS}_{2}$. Phys Rev Lett 87:196803. doi:10.1103/PhysRevLett.87.196803

41. Tsai C, Chan K, Nørskov JK, Abild-Pedersen F (2015) Rational design of $\mathrm{MoS}_{2}$ catalysts: tuning the structure and activity via transition metal doping. Catal Sci Technol 5:246-253. doi:10.1039/C4CY01162G

42. Tsai C, Chan K, Nørskov JK, Abild-Pedersen F (2014) Understanding the reactivity of layered transition-metal sulfides: a single electronic descriptor for structure and adsorption. J Phys Chem Lett 5:3884-3889. doi:10.1021/jz5020532

43. Wang H, Tsai C, Kong D et al (2015) Transition-metal doped edge sites in vertically aligned $\mathrm{MoS}_{2}$ catalysts for enhanced hydrogen evolution. Nano Res 8:566-575. doi:10.1007/s12274-014-0677-7

44. Friebel D, Louie MW, Bajdich M et al (2015) Identification of highly active $\mathrm{Fe}$ sites in $(\mathrm{Ni}, \mathrm{Fe}) \mathrm{OOH}$ for electrocatalytic water splitting. J Am Chem Soc 137:1305-1313. doi:10.1021/ ja511559d

45. Burke MS, Kast MG, Trotochaud L et al (2015) Cobalt-iron (oxy) hydroxide oxygen evolution electrocatalysts: the role of structure and composition on activity, stability, and mechanism. J Am Chem Soc 137:3638-3648. doi:10.1021/jacs.5b00281

46. Zhang B, Zheng X, Voznyy O et al (2016) Homogeneously dispersed, multimetal oxygen-evolving catalysts. Science. doi:10.1126/science.aaf 1525

47. McCrory CCL, Jung S, Ferrer IM et al (2015) Benchmarking HER and OER electrocatalysts for solar water splitting devices. J Am Chem Soc 137:4347-4357. doi:10.1021/ja510442p

48. McCrory CCL, Jung S, Peters JC, Jaramillo TF (2013) Benchmarking heterogeneous electrocatalysts for the oxygen evolution reaction. J Am Chem Soc 135:16977-16987. doi:10.1021/ ja407115p

49. Nørskov JK, Rossmeisl J, Logadottir A et al (2004) Origin of the overpotential for oxygen reduction at a fuel-cell cathode. J Phys Chem B 108:17886-17892. doi:10.1021/jp047349j

50. Rossmeisl J, Qu Z-W, Zhu H et al (2007) Electrolysis of water on oxide surfaces. J Electroanal Chem 607:83-89. doi:10.1016/j. jelechem.2006.11.008 
51. Abild-Pedersen F, Greeley J, Studt F et al (2007) Scaling properties of adsorption energies for hydrogen-containing molecules on transition-metal surfaces. Phys Rev Lett 99:016105. doi:10.1103/ PhysRevLett.99.016105

52. Fernández EM, Moses PG, Toftelund A et al (2008) Scaling relationships for adsorption energies on transition metal oxide, sulfide, and nitride surfaces. Angew Chem Int Ed Engl 47:46834686. doi:10.1002/anie.200705739

53. Greeley J, Stephens IEL, Bondarenko AS et al (2009) Alloys of platinum and early transition metals as oxygen reduction electrocatalysts. Nat Chem 1:552-556. doi:10.1038/nchem.367

54. Viswanathan V, Hansen HA, Rossmeisl J, Nørskov JK (2012) Universality in oxygen reduction electrocatalysis on metal surfaces. ACS Catal 2:1654-1660. doi:10.1021/cs300227s

55. Giannozzi P, Baroni S, Bonini N et al (2009) QUANTUM ESPRESSO: a modular and open-source software project for quantum simulations of materials. J Phys Condens Matter 21:395502. doi:10.1088/0953-8984/21/39/395502

56. Atomic Simulation Environment (ASE) Center for Atomic Scale Material Design (CAMD), Technical University of Denmark, Lyngby. https://wiki.fysik.dtu.dk/ase

57. Wellendorff J, Lundgaard KT, Møgelhøj A et al (2012) Density functionals for surface science: exchange-correlation model development with Bayesian error estimation. Phys Rev B 85:235149. doi:10.1103/PhysRevB.85.235149
58. Kresse G (1996) Efficient iterative schemes for ab initio totalenergy calculations using a plane-wave basis set. Phys Rev B 54:11169-11186. doi:10.1103/PhysRevB.54.11169

59. Kresse G, Furthmüller J (1996) Efficiency of ab-initio total energy calculations for metals and semiconductors using a plane-wave basis set. Comput Mater Sci 6:15-50. doi:10.1016/0927-0256(96)00008-0

60. Dudarev SL, Savrasov SY, Humphreys CJ, Sutton AP (1998) Electron-energy-loss spectra and the structural stability of nickel oxide: an LSDA + U study. Phys Rev B 57:1505-1509. doi:10.1103/PhysRevB.57.1505

61. Ng JWD, García-Melchor M, Bajdich M et al (2016) Goldsupported cerium-doped $\mathrm{NiOx}$ catalysts for water oxidation. Nat Energy 1:16053. doi:10.1038/nenergy.2016.53

62. Siahrostami S, Vojvodic A (2015) Influence of adsorbed water on the oxygen evolution reaction on oxides. J Phys Chem C 119:1032-1037. doi:10.1021/jp508932x

63. Koper MTM (2013) Theory of multiple proton-electron transfer reactions and its implications for electrocatalysis. Chem Sci 4:2710. doi:10.1039/c3sc50205h

64. Man IC, Su H-Y, Calle-Vallejo F et al (2011) Universality in oxygen evolution electrocatalysis on oxide surfaces. ChemCatChem 3:1159-1165. doi: $10.1002 /$ cctc. 201000397 